Pirineos, 149-150: 3 a 20, JACA; 1997

\title{
INFLUENCIA DE LA LITOLOGÍA EN LOS PROCESOS DE EROSIÓN EN BADLANDS. LOS CASOS DE ANNA (VALENCIA) Y PETRER (ALICANTE)
}

\author{
A. CERDÀ \\ Departament de Geografia. Universitat de València. Apartado 22060, 46080 València, España. \\ Tel. 34638642 37. Fax. 34638642 49. E-mail: acerdn@uv.es \\ Centro de Investigaciones sobre Desertificación-CIDE (CSIC, Universitat de València, Generalitat \\ Valenciana). Cami de la Marjal, s/n, 46470 Albal, València, España
}

RESUUMEN.- A partir de experimentos con lluvia simulada se estudia el comportamiento hidrológico y erosivo de superficies degradadas (badlands) desarrolladas sobre dos litologias distintas: margas y arcillas. En las arcillas del Keuper el encharcamiento ( $\left.4^{\prime} 07^{\prime \prime}\right)$ y la escorrentía ( $6^{\prime} 36^{\prime \prime}$ ) es retrasada, las tasas de infiltración son elevadas $\left(38,63 \mathrm{~mm} \mathrm{~h}^{-1}\right), y$ los coeficientes de escorrentía (11,2\% tras 30 minutos $y$ $19,4 \%$ tras 60 minutos) y las tasas de erosión $\left(13 \mathrm{~g} \mathrm{~m}^{2} \mathrm{~h}^{-1}\right)$ reducidas. En cambio, sobre margas Cretácicas, el encharcamiento ( $\left.1^{\prime} 37^{\prime \prime}\right)$ y la escorrentía superficial $\left(2^{\prime}\right.$ $\left.52^{\prime \prime}\right)$ se produce casi inmediatamente, las tasas de infiltración son muy bajas (11,2 $\left.m m^{-1}\right)$, y los coeficientes de escorrentía $(64,7 \%$ tras 30 minutos y $72,2 \%$ tras 60 minutos) y las tasas de erosión son elevadísimas $\left(2.198 \mathrm{~g} \mathrm{~m}^{2} \mathrm{~h}^{-1}\right)$. La actividad de la arroyada superficial en los badlands sobre margas hace que las posibilidades de regeneración de los suelos y la cubierta vegetal sean reducidas.

ABSTRACT.- By means of experiments with simulated rainfall the hydrological and erosional behaviour of degraded surfaces (badlands) developed on two different parent materials (marls and clays) is studied. On the Keuper clays, the ponding $\left(4^{\prime} 07^{\prime \prime}\right)$ and runoff $\left(6^{\prime} 36^{\prime \prime}\right)$ initiation is delayed, the infiltration rates (38.63 $\left.\mathrm{mmh}^{-1}\right)$ are high, and the runoff coefficient (11.2\% after 30 minutes and $19.4 \%$ after 60 minutes) and the erosion rates $\left(13 \mathrm{~g} \mathrm{~m}^{2} \mathrm{~h}^{-1}\right)$ are lowv. On the contrary, over Cretaceous marls, the ponding ( 1 '37") and the surface runoff (2' 52") initiation is very rapid, the infiltration rates are low $\left(11.2 \mathrm{~mm} \mathrm{~h}^{-1}\right)$, and the runoff coefficient (64.7\% after 30 minutes and $72.2 \%$ after 60 minutes) and the erosion rates (2,198 $\left.\mathrm{g} \mathrm{m}^{2} h^{-1}\right)$ are very high. The activity of the surface runoff on the badlands over marls results in very low possibilities of soil and vegetation cover regeneration.

RÉSUMÉ.- Sur la base de différentes expériments de pluie simulée l'auteur étudie le comportement hydrologique et érosif de deux badlands établis su deux types de 
roche-mère: marnes et argile. Sur les argiles du Keuper l'accumulation d'eau (4'05") et l'écoulement $\left(6^{\prime} 36^{\prime \prime}\right)$ sont retardés, les taux d'infiltration son elevés $(38,63$ $\left.m m h^{-1}\right)$ et les coefficients d'écoulement (11,2\% après 30 minutes et 19,4\% après 60 minutes) et les taux d'érosion sont réduits. Par contre, sur les marnes de Cretacé, l'accumulation d'eau (1'37") et l'ecoulement superficielle (2' 52") ont lieu presque immédiatement, les taux d'infiltration restent très basse $\left(11,2 \mathrm{mmh}^{-1}\right)$ et les coefficients d'écoulement (64,7\% après 30 minutes et $72,2 \%$ après 60 minutes) et les taux d'érosion deviennent très élevés $\left(2198 \mathrm{~g} \mathrm{~m}^{2} \mathrm{~h}^{-1}\right)$. Le ravinnement superficiel très actif sur les badlands reduit fortement les possibilités de régeneration des sols et la restoration du couvert végétal.

Key-words: Badlands, parent material, clays, marls, hydrology, erosion, simulated rainfall.

\section{Introducción}

El roquedo, junto con el clima, determina los procesos geomorfológicos superficiales debido al control que ejerce sobre los procesos de meteorización física, química y biológica, la formación y desarrollo de los suelos y el modelado de las laderas (SUMMERFIELD, 1991; LÓPEZ BERMÚDEZ et al., 1992) . En las zonas degradadas la influencia de la litología sobre los procesos de erosión es aún más importante por la escasez de vegetación que amortigüe el impacto de las gotas de lluvia y la arroyada superficial (JORGENSEN \& GARDNER, 1987). Esto da lugar a un control directo de las características del roquedo sobre la capacidad de infiltración (BERNDTSSON et al., 1985) y la erodibilidad de los suelos (SANROQUE et al., 1990).

En los ecosistemas semiáridos son abundantes las zonas de badlands en las que los suelos consisten en una capa de regolita de pocos centímetros de profundidad, las laderas están surcadas por regueroș, en ocasiones se generan pequeños tubos (piping), la vegetación es insignificante y los valles presentan cárcavas de grandes dimensiones (BRYAN \& YAIR, 1982; CLOTĖT et al., 1987; ARNÁEZ, 1988; CAMPBELL, 1989; HOWARD, 1994). Estos ambientes se desarrollan sobre litologías blandas como son las margas, arcillas, sedimentos cuaternarios, etc., donde los procesos erosivos son mucho más activos.

Los estudios realizados hasta el momento en los badlands han incidido en las morfologías superficiales de los suelos o laderas y los procesos erosivos e hidrológicos, sin tener en cuenta la diversidad inducida por el roquedo en estos ambientes. En este trabajo se investiga la influencia del roquedo sobre los procesos de erosión en badlands. Para ello se han seleccionado dos de los substratos litológicos blandos más abundantes en el sudeste peninsular -las arcillas y las margas-con el fin de comparar la respuesta ante la lluvia de los suelos desarrollados a partir de rocas madres distintas, pero que presentan 
morfologías superficiales similares (encostramiento, grietas, regueros, inexistencia de vegetación, etc.). Con este fin se han llevado a cabo experimentos con lluvia simulada, lo que permite realizar mediciones de elevada precisión debido al control de distintos factores (precipitación, humedad previa, cambios en las costras superficiales y en las grietas, etc.), además de acelerar la toma de datos y reducir los costes de la investigación. En la comparación de dos situaciones distintas - como aquí se presentan - la posibilidad de repetir y reproducir experimentos en las mismas condiciones es fundamental para conocer las diferencias entre ellas. También se ofrece una amplia revisión bibliográfica de un tema, - los badlands- de gran interés para los geomorfólogos, pero cada vez más estudiado por los botánicos, edafólogos e hidrólogos.

El objetivo de este artículo es proporcionar datos obtenidos con la misma metodología de dos zonas de badlands con distintas litologías. Ello permitirá profundizar en los procesos geomorfológicos que actúan en estos ambientes y conocer el comportamiento de estos suelos ante la lluvia.

\section{Materiales y Métodos}

\subsection{Zonas de estudio}

Para el estudio se seleccionaron dos zonas acarcavadas en el sudeste de la Península Ibérica: Petrer y Anna (Figura 1). En Petrer, al norte de la provincia de Alicante, el roquedo son margas cretácicas y existen suelos desnudos (badlands) tanto en las laderas norte como en las sur (Figura 2). Todos ellos están relacionados con la incisión del anterior valle pleistoceno debido al diapirismo de la zona. El abancalamiento y posterior abandono del fondo de valle ha favorecido la formación de cárcavas en los últimos 20 años (RODRÍGUEZ, 1992). Las condiciones climatológicas se caracterizan por la baja precipitación media anual, la cual oscila entre 296 y $339 \mathrm{~mm}$ según autores (GEIGER, 1970; PAYÀ \& CERDÀ, 1992). La temperatura media anual es de $16^{\circ} \mathrm{C}$, con un máximo de $25,7^{\circ} \mathrm{C}$ en agosto y $11,4{ }^{\circ} \mathrm{C}$ en enero. La media anual de días de lluvia es de 33 .

En Anna, al sur de la provincia de Valencia, la roca madre son arcillas del Keuper, existiendo suelos desnudos (badlands) en las partes bajas de las laderas norte y sur (Figura 3), lo que está directamente relacionado con la incisión del cauce debido al diapirismo propio de la zona. También aquí el desarrollo de los badlands es mayor en la ladera sur como consecuencia del mayor estrés hídrico. Las precipitaciones son mayores en Anna $(548 \mathrm{~mm})$ que en Petrer, a 
PIRINEOS 149-150

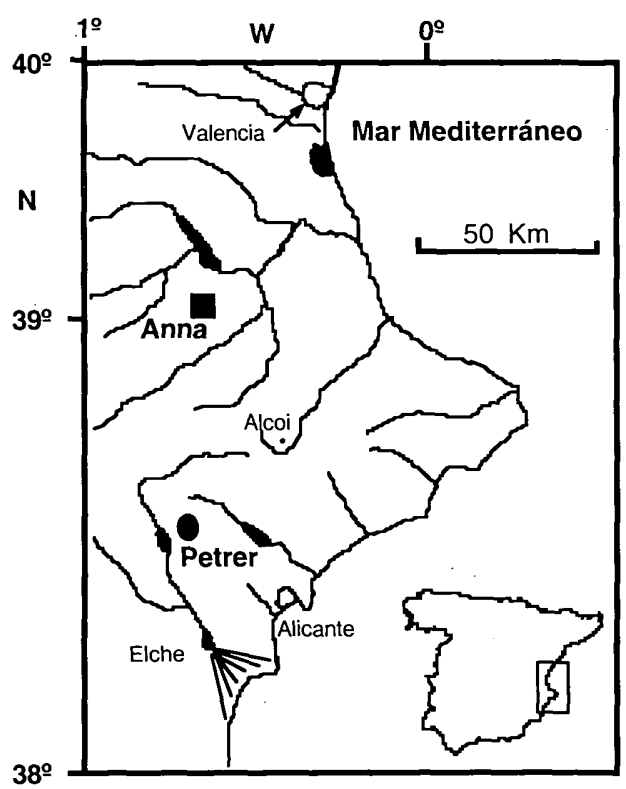

Figura 1. Localización de las zonas de estudio. Valencia, Alicante Location of the study sites. Valencia-Alicante

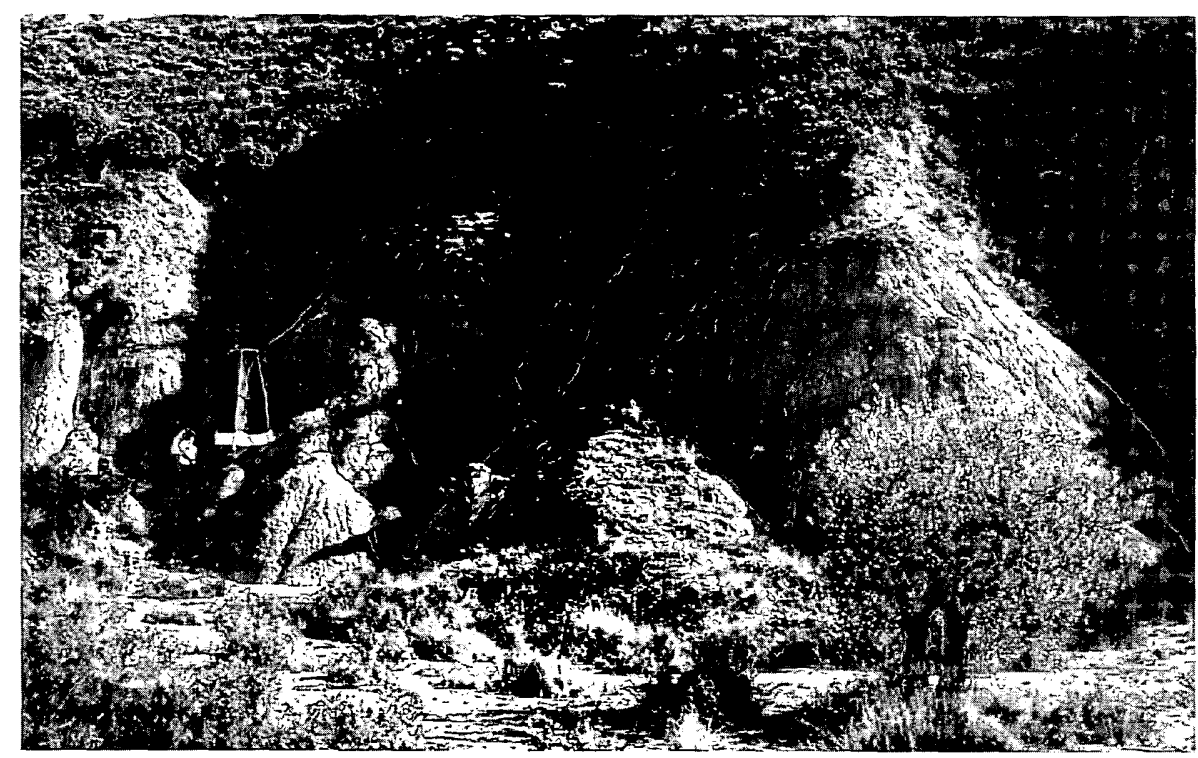

Figura 2. Vista de la zona de estudio de Petrer. Alicante. View of the study site of Petrer. Alicante.

6 


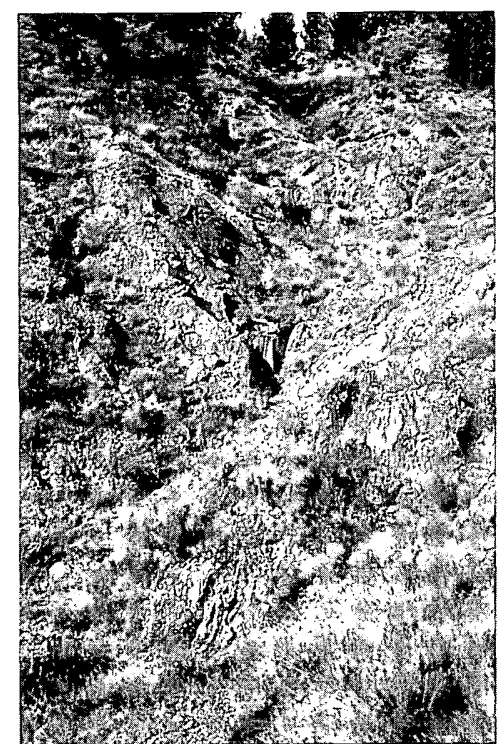

Figura 3. Vista de la zona de estudio de Anna, Valencia View of the study site of Anna, Valencia

pesar de lo cual los suelos de las partes bajas de las laderas no presentan cubierta vegetal. La temperatura media anual es de $17,7^{\circ} \mathrm{C}$, con un máximo de $26,8^{\circ} \mathrm{C}$ en agosto y $10,2^{\circ} \mathrm{C}$ en enero. El número de días de lluvia es 55 .

Estudios previos realizados en ambas cuencas experimentales han hecho especial hincapié en la morfología de las laderas y superficies, y los procesos de infiltración, generación de escorrentía y erosión.

\subsection{Mediciones realizadas}

En ambas zonas de estudio, Petrer y Anna, se seleccionaron cinco parcelas de $0,24 \mathrm{~m}^{2}$ en laderas de exposición sur con el fin de estudiar el proceso de erosión a escala entre regueros. Los experimentos se llevaron a cabo con un simulador de lluvia portátil (CERDÀ et al., 1997). Las mediciones se realizaron en el verano de 1991 cuando la humedad del suelo era muy baja (3-6 \% en superficie). La duración de cada chaparrón fue de 60 minutos a una intensidad de $55 \mathrm{~mm} \mathrm{~h}^{-1}$, lo que permite la comparación entre las distintas parcelas y zonas de estudios. El periodo de retorno de estos chaparrones es de 7-8 años en la zona de estudio de Anna y de 10-12 en la de Petrer (ELÍAS \& RUIZ, 1979).

En todos los suelos la vegetación es inexistente y las pendientes elevadas (mayor de $20^{\circ}$ ). Las parcelas tienen una longitud de $55 \mathrm{~cm}$, y se tomó mues- 
tra de caudal cada 30 ó 60 segundos para conocer la curva de escorrentía completa. Al menos tres veces se tomaron muestras para determinar la concentración de sedimentos y las tasas de erosión. En tres casos la concentración de sedimentos de la escorrentía se muestreó a intervalos de un minuto (todo el caudal) con el fin de conocer con mayor precisión los cambios temporales de la erosionabilidad de los suelos. Los 40 minutos de duración de los experimentos fueron suficientes para alcanzar las tasas de infiltración final estable. El tiempo de encharcamiento $(t p)$ se determinó de forma visual $(40 \%$ de la superficie encharcada) y el inicio de la escorrentía ( $t r)$ cuando ésta se inicia en el colector.

La humedad previa del suelo se midió por el método gravimétrico y la concentración de sedimentos por desecación. La materia orgánica por el método de Walkley-Black, la textura por el de sedimentación discontinua (clasificación USDA), el carbonato cálcico por Calcimetría de Bernard y la densidad aparente por medio de muestras inalteradas tomadas con anillo.

Las parcelas estudiadas corresponden a la morfología más extendida en ambas zonas de estudio. En el caso de Petrer —badlands sobre margas- las superficies presentan bajas cubiertas de fragmentos de rocas, nulas cubiertas vegetales y costras superficiales muy densas con un elevado índice de agrietamiento. Las grietas alcanzan anchuras de hasta $1 \mathrm{~cm}$ (de 4 a $10 \mathrm{~mm}$ ) y forman polígonos de 5-10 $\mathrm{cm}$ de anchura. Estas grietas alcanzan profundidades de 3-4 cm y en ocasiones están conectadas con la red de fracturas de la roca madre. También existe una segunda red de grietas de menor anchura $(1 \mathrm{~mm})$ y profundidad (1-3 $\mathrm{mm}$ ) aunque no llegan a formar una red de polígonos permanente como las grietas de mayor tamaño (Figura 4).

En Anna -badlands sobre arcillas del Keuper- las grietas alcanzan anchuras de hasta $2-3 \mathrm{~cm}$ que no llegan a formar polígonos (Figura 5). Estas grietas presentan profundidades de hasta $8-9 \mathrm{~cm}$ (mayores cuanto más anchas) y que no parecen conectadas con la red de fracturas de la roca madre. Tampoco existe una segunda red de grietas de menor anchura como ocurre en los badlands estudiados en Petrer.

En general, los suelos de ambas zonas de estudio se caracterizan por el reducido contenido de materia orgánica, la textura fina y la elevada densidad aparente (Tablas 1 y 2). En concreto, en Petrer la materia orgánica en los suelos es insignificante, tanto en superficie $(0-2 \mathrm{~cm}, 0,22 \%)$ como por debajo de la superficie (4-6 cm, 0,16\%), mientras que en Anna, los valores son ligeramente mayores $(0-2 \mathrm{~cm}, 0,58 \% ; 4-6 \mathrm{~cm}, 0,42 \%)$. La textura es arcillosa en Anna y arcillo-limosa o franco-arcillo-limoso en Petrer. La diferencia entre los dos horizontes analizados es insignificante en Petrer, lo que refleja el nulo desarrollo de los suelos, mientras que en Anna el suelo es ligeramente más arcilloso en profundidad. 
INFLUENCIA DE LA LITOLOGÍA EN LOS PROCESOS DE EROSIÓN EN BADLANDS

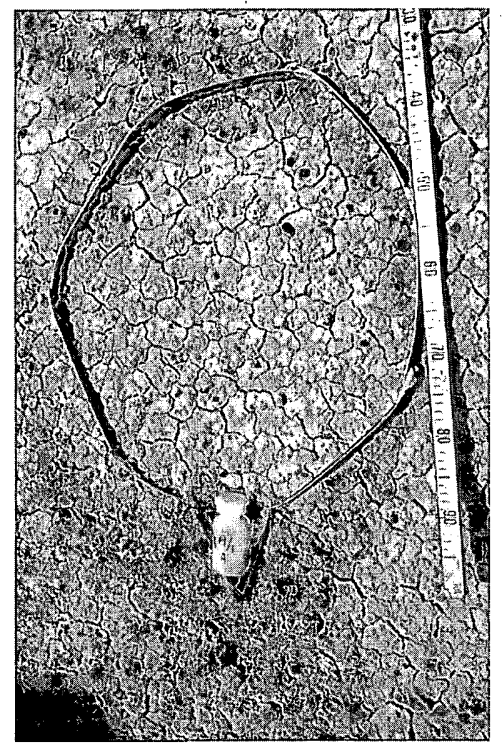

Figura 4. Parcela de Petrer. Badlands sobre margas cretácicas. Plot of Petrer. Badland on Cretaceous marls.

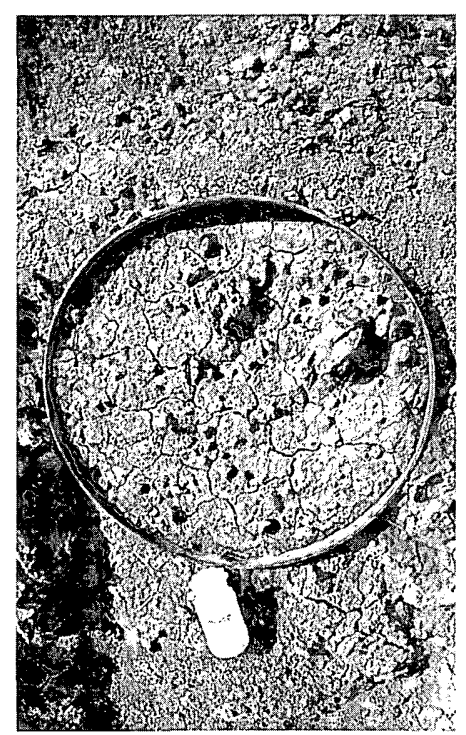

Figura 5. Parcela de Anna. Badlands sobre arcillas del Keuper. Plot of Anna. Badland on Keuper clays. 
PIRINEOS 149-150

\begin{tabular}{|crrrrrr|}
\hline $\begin{array}{c}\text { Parcelas } \\
(0-2 \mathrm{~cm})\end{array}$ & $\begin{array}{c}\mathrm{MO} \\
(\%)\end{array}$ & $\begin{array}{c}\mathrm{CaCo}_{3} \\
(\%)\end{array}$ & $\begin{array}{c}\text { Densidad } \\
\left(\mathrm{g} \mathrm{cm}^{-3}\right)\end{array}$ & $\begin{array}{c}\text { Arena } \\
(\%)\end{array}$ & $\begin{array}{r}\text { Limo } \\
(\%)\end{array}$ & \multicolumn{1}{c|}{$\begin{array}{c}\text { Arcilla } \\
(\%)\end{array}$} \\
\hline ANNA & & & & & & \\
A & 0,61 & 3,12 & 1,10 & 10,10 & 36,55 & 53,35 \\
B & 0,36 & 2,02 & 1,16 & 7,76 & 28,53 & 63,71 \\
C & 0,46 & 2,69 & 1,20 & 6,93 & 40,35 & 52,72 \\
D & 0,42 & 2,15 & 1,18 & 8,63 & 32,22 & 59,15 \\
E & 1,03 & 3,25 & 1,16 & 8,63 & 32,22 & 59,15 \\
Media & 0,58 & 2,65 & 1,16 & 8,41 & 33,97 & 57,62 \\
Std & 0,24 & 0,50 & 0,03 & 1,06 & 4,08 & 4,10 \\
Cv (\%) & 41,93 & 18,74 & 2,89 & 12,55 & 12,00 & 7,11 \\
\hline PETRER & & & & & & \\
A & 0,10 & 71,32 & 1,52 & 8,36 & 51,23 & 40,41 \\
B & 0,10 & 69,25 & 1,49 & 7,39 & 60,29 & 32,32 \\
C & 0,34 & 70,25 & 1,51 & 9,26 & 41,02 & 49,72 \\
D & 0,25 & 69,54 & 1,45 & 8,17 & 46,95 & 44,88 \\
E & 0,31 & 68,25 & 1,63 & 7,69 & 58,32 & 33,99 \\
Media & 0,22 & 69,72 & 1,52 & 8,17 & 51,56 & 40,26 \\
Std & 0,10 & 1,03 & 0,06 & 0,64 & 7,13 & 6,53 \\
Cv (\%) & 46,44 & 1,47 & 3,95 & 7,86 & 13,83 & 16,22 \\
\hline
\end{tabular}

Tabla 1. Principales características de los suelos. Materia orgánica (MO), carbonato cálcico, densidad aparente y textura en las superficies de cada una de las parcelas estudiadas. Profundidad entre 0 y $2 \mathrm{~cm}$.

Main soil characteristics. Organic matter (MO), calcium carbonate, bulk density and grain size at the surface layer of the studied plots. Depth between 0 and $2 \mathrm{~cm}$.

La densidad aparente es muy alta en Petrer: $1,52 \mathrm{~g} \mathrm{~cm}^{-3}$ en superficie y 1,57 $\mathrm{g} \mathrm{cm}^{-3}$ a una profundidad entre 4 y $6 \mathrm{~cm}$. A estas mismas profundidades encontramos valores mucho menores en el caso de Anna: 1,16 y $1,19 \mathrm{~g} \mathrm{~cm}^{-3}$ respectivamente. Además de las pequeñas diferencias en materia orgánica y textura, y las mayores en densidad aparente de los suelos, la diferencia más importante se ha encontrado en el contenido en carbonato cálcico. El origen calcáreo de las margas hace que los niveles de carbonato cálcico alcancen el $70 \%$, mientras que en las arcillas del Keuper es menor al $3 \%$ por término medio.

La variabilidad espacial de los parámetros comentados (Tabla 1) es reducida con la excepción de la materia orgánica. Ello es debido a que los suelos de los badlands están muy degradados y no desarrollan cubiertas vegetales estables que a su vez favorezcan el desarrollo de zonas con suelos distintos que den lugar a mayores diferencias espaciales.

La estructura de los suelos en Petrer es débil, de consistencia dura en seco y friable/muy friable en húmedo, y muy adherente y plástica en mojado; mientras que en Anna la estructura es moderada, e incluso fuerte en algunos casos, 
INFLUENCIA DE LA LITOLOGÍA EN LOS PROCESOS DE EROSIÓN EN BADLANDS

\begin{tabular}{|ccrcrrr|}
\hline $\begin{array}{c}\text { Parcelas } \\
(4-6 \mathrm{~cm})\end{array}$ & $\begin{array}{c}\mathrm{MO} \\
(\%)\end{array}$ & $\begin{array}{c}\mathrm{CaCo}_{3} \\
(\%)\end{array}$ & $\begin{array}{c}\text { Densidad } \\
\left(\mathrm{g} \mathrm{cm}^{-3}\right)\end{array}$ & $\begin{array}{c}\text { Arena } \\
(\%)\end{array}$ & $\begin{array}{c}\text { Limo } \\
(\%)\end{array}$ & $\begin{array}{c}\text { Arcilla } \\
(\%)\end{array}$ \\
\hline ANNA & & & & & & \\
A & 0,12 & 2,15 & 1,12 & 5,11 & 30,33 & 64,56 \\
B & 1,35 & 3,09 & 1,20 & 4,17 & 33,26 & 62,57 \\
C & 0,12 & 2,14 & 1,22 & 4,72 & 36,92 & 58,36 \\
D & 0,31 & 3,05 & 1,19 & 4,67 & 32,99 & 62,34 \\
E & 0,21 & 1,82 & 1,24 & 5,41 & 26,92 & 67,67 \\
Media & 0,42 & 2,45 & 1,19 & 4,82 & 32,08 & 63,10 \\
Std & 0,47 & 0,52 & 0,04 & 0,42 & 3,33 & 3,04 \\
Cv (\%) & 111,9 & 21,23 & 3,42 & 8,75 & 10,37 & 4,82 \\
\hline PETRER & & & & & & \\
A & 0,10 & 70,25 & 1,53 & 6,99 & 52,49 & 40,52 \\
B & 0,10 & 70,01 & 1,55 & 6,98 & 53,29 & 39,73 \\
C & 0,21 & 72,26 & 1,60 & 5,96 & 54,32 & 39,72 \\
D & 0,13 & 68,20 & 1,52 & 7,48 & 51,69 & 40,83 \\
E & 0,26 & 69,25 & 1,63 & 7,57 & 52,31 & 40,12 \\
Media & 0,16 & 69,99 & 1,57 & 7,00 & 52,82 & 40,18 \\
Std & 0,06 & 1,34 & 0,04 & 0,57 & 0,91 & 0,44 \\
Cv (\%) & 40,12 & 1,91 & 2,70 & 8,18 & 1,72 & 1,09 \\
\hline
\end{tabular}

Tabla 2. Principales características de los suelos. Materia orgánica (MO), textura, densidad aparente, carbonato cálcico y pendiente en las superficies de cada una de las parcelas estudiadas. Profundidad entre 4 y $6 \mathrm{~cm}$.

Main soil characteristics. Organic matter (MO), calcium carbonate, bulk density and grain size at the surface layer of the studied plots. Depth between 4 and $6 \mathrm{~cm}$.

de consistencia dura en seco y friable en húmedo, y muy adherente y muy plástica en mojado. Aunque los suelos pueden se clasificados como regosoles, en realidad en ambos casos consisten en un espesor de 1-5 cm de regolita en Petrer ( $2,6 \mathrm{~cm}$ de media) y $10-26 \mathrm{~cm}$ (17 cm de media) en Anna. La pendiente en algunos casos es muy elevada: $27,4^{\circ}$ y $33,8^{\circ}$ respectivamente en Anna y Petrer. La cubierta de plantas es insignificante, aunque en Anna existe una parcela con una cubierta del $20 \%$, lo que es fruto de la mayor pluviosidad de la zona.

\section{Resultados}

\subsection{Respuesta hidrológica}

La humedad previa antes de los experimentos era muy reducida en ambas zonas de estudio (Tabla 3). Los valores medios no superan el $2 \%$ en ningún 
PIRINEOS 149-150

\begin{tabular}{|ccccccc|}
\hline $\begin{array}{c}\text { Parcelas } \\
\left({ }^{\circ}\right)\end{array}$ & $\begin{array}{c}p e n \\
(\%)\end{array}$ & $\begin{array}{c}p l \\
(\mathrm{~cm})\end{array}$ & $\begin{array}{c}p s \\
(\%)\end{array}$ & $\begin{array}{c}h s \\
(\%)\end{array}$ & $\begin{array}{c}h s s \\
(\%)\end{array}$ & $h m$ \\
\hline ANNA & & & & & & \\
A & 25 & 10 & 15 & 0,1 & 2,0 & 1,1 \\
B & 22 & 5 & 26 & 0,8 & 1,6 & 1,2 \\
C & 22 & 2 & 10 & 0,5 & 2,3 & 1,4 \\
D & 44 & 0 & 15 & 1,0 & 2,1 & 1,6 \\
E & 24 & 20 & 19 & 0,2 & 1,2 & 0,7 \\
Media & 27,40 & 7,40 & 17,00 & 0,53 & 1,83 & 1,18 \\
Std & 8,38 & 7,14 & 5,33 & 0,33 & 0,40 & 0,29 \\
Cv (\%) & 30,59 & 96,54 & 31,35 & 61,55 & 21,82 & 24,81 \\
\hline PETRER & & & & & & \\
A & 37 & 0 & 1 & 1,3 & 2,7 & 2,0 \\
B & 30 & 0 & 3 & 1,8 & 2,5 & 2,1 \\
C & 33 & 2 & 5 & 1,3 & 2,6 & 1,9 \\
D & 34 & 0 & 2 & 1,3 & 2,6 & 1,9 \\
E & 30 & 0 & 2 & 1,0 & 2,1 & 1,6 \\
Media & 32,80 & 0,40 & 2,60 & 1,33 & 2,49 & 1,91 \\
Std & 2,64 & 0,80 & 1,36 & 0,27 & 0,20 & 0,20 \\
Cv (\%) & 8,04 & 200,00 & 52,17 & 20,20 & 8,19 & 10,23 \\
\hline
\end{tabular}

Tabla 3. Pendiente (pen), cubierta de plantas (pl), profundidad del suelo (ps), humedad superficial $(0-2 \mathrm{~cm})(\mathrm{hs})$, humedad subsuperficial $(4-6 \mathrm{~cm})(\mathrm{hss})$ y media entre hs y hss $(\mathrm{hm})$. Angle slope (pen), plant cover ( $p l)$, soil depth ( $p s)$, surface soil moisture $(0-2 \mathrm{~cm})(\mathrm{hs})$, subsurface soil moisture $(4-6 \mathrm{~cm})(\mathrm{hss})$ and average between hs and hss $(\mathrm{hm})$.

caso. A pesar de ello, la respuesta de los suelos ante la lluvia fueron muy distintas en Petrer y Anna (Tabla 4).

En general, la lluvia origina cambios muy importantes en las superficies de los badlands, y además estos cambios se producen rápidamente. En el caso de Petrer los cambios fueron mucho más rápidos que en Anna. Allí, el encharcamiento del suelo se produjo casi inmediatamente $\left(1^{\prime} 37^{\prime \prime}\right)$, oscilando entre $1^{\prime}$ y $2^{\prime}$. En Anna osciló entre 2' 20" y 6' 24", con 4' 07" de valor medio. La escorrentía superficial apareció tras $1^{\prime} 15^{\prime \prime}$ en Petrer y $2^{\prime} 19^{\prime \prime}$ en Anna. Concretamente, la escorrentía se produjo después de $2^{\prime} 52^{\prime \prime}\left(1^{\prime} 50^{\prime \prime}-5^{\prime}\right)$ en Petrer y $6^{\prime} 36^{\prime \prime}\left(3^{\prime} 1^{\prime \prime}-10^{\prime \prime} 54^{\prime \prime}\right)$ en Anna. Tras la escorrentía en superficie, se produjo la medición de la escorrentía en el colector con un tiempo de retraso escaso (40" en Petrer y 1' en Anna). Esta rápida transmisión de la escorrentía a pesar de las grietas se debe a la falta de hojarasca y plantas. El cierre de las grietas fue muy retrasado en Anna, 15' 36', oscilando entre $6^{\prime}$ y $22^{\prime}$. En cambio, en Petrer fue de $8^{\prime} 21^{\prime \prime}$, oscilando entre $2^{\prime}$ y $12^{\prime \prime}$.

La escorrentía se produjo con mayor rapidez y con volúmenes de precipitación muy reducidos en el caso de Petrer: $2,57 \mathrm{~mm}(1,38-4,58 \mathrm{~mm})$ y bas- 
INFLUENCIA DE LA LITOLOGÍA EN LOS PROCESOS DE EROSIÓN EN BADLANDS

\begin{tabular}{|c|c|c|c|c|c|c|}
\hline Parcelas & $t p$ & te & $\operatorname{tg} c$ & tes & tef & $\begin{array}{c}\mathrm{Pe} \\
(\mathrm{mm})\end{array}$ \\
\hline \multicolumn{7}{|l|}{ ANNA } \\
\hline A & $3^{\prime} 09^{\prime \prime}$ & $7^{\prime} 07^{\prime \prime}$ & $6^{\prime} 00^{\prime \prime}$ & $8^{\prime} 00^{\prime \prime}$ & $0^{\prime} 41^{\prime \prime}$ & 6,52 \\
\hline B & $6^{\prime} 24^{\prime \prime}$ & $10^{\prime} 54^{\prime \prime}$ & $21^{\prime} 00^{\prime \prime}$ & $16^{\prime} 00^{\prime \prime}$ & $0^{\prime} 33^{\prime \prime}$ & 9,99 \\
\hline $\mathrm{C}$ & $3^{\prime} 10^{\prime \prime}$ & $3^{\prime} 50^{\prime \prime}$ & $15^{\prime} 00^{\prime \prime}$ & $5^{\prime} 00^{\prime \prime}$ & $0^{\prime} 66^{\prime \prime}$ & 3,51 \\
\hline $\mathrm{D}$ & $5^{\prime} 30^{\prime \prime}$ & $8^{\prime} 00^{\prime \prime}$ & $22^{\prime} 00^{\prime \prime}$ & $10^{\prime} 00^{\prime \prime}$ & $0^{\prime} 50^{\prime \prime}$ & 7,33 \\
\hline E & $2^{\prime} 20^{\prime \prime}$ & $3^{\prime} 10^{\prime \prime}$ & $14^{\prime} 00^{\prime \prime}$ & $4^{\prime} 00^{\prime \prime}$ & $1^{\prime} 48^{\prime \prime}$ & 2,90 \\
\hline Media & $4^{\prime} 07^{\prime \prime}$ & $6^{\prime} 36^{\prime \prime}$ & $15^{\prime} 36^{\prime}$ & $8^{\prime} 36^{\prime \prime}$ & $0^{\prime} 52^{\prime \prime}$ & 6,05 \\
\hline \multicolumn{7}{|l|}{ PETRER } \\
\hline A & $1^{\prime} 30^{\prime \prime}$ & $3^{\prime} 00^{\prime \prime}$ & $7^{\prime} 05^{\prime \prime}$ & $3^{\prime} 30^{\prime \prime}$ & $1^{\prime} 00^{\prime \prime}$ & 2,75 \\
\hline B & $2^{\prime} 00^{\prime \prime}$ & $2^{\prime} 30^{\prime \prime}$ & $2^{\prime} 00^{\prime \prime}$ & $4^{\prime} 00^{\prime \prime}$ & $0^{\prime} 50^{\prime \prime}$ & 2,29 \\
\hline C & $1^{\prime} 00^{\prime \prime}$ & $1^{\prime} 50^{\prime \prime}$ & $10^{\prime} 00^{\prime \prime}$ & $2^{\prime} 20^{\prime \prime}$ & $1^{\prime} 00^{\prime \prime}$ & 1,38 \\
\hline $\mathrm{D}$ & $1^{\prime} 35^{\prime \prime}$ & $2^{\prime} 02^{\prime \prime}$ & $10^{\prime} 41^{\prime \prime}$ & $2^{\prime} 20^{\prime \prime}$ & $1^{\prime} 00^{\prime \prime}$ & 1,86 \\
\hline$E$ & $2^{\prime} 00^{\prime \prime}$ & $5^{\prime} 00^{\prime \prime}$ & $12^{\prime} 00^{\prime \prime}$ & $5^{\prime} 30^{\prime \prime}$ & $2^{\prime} 00^{\prime \prime}$ & 4,58 \\
\hline Media & $1^{\prime} 37^{\prime \prime}$ & $2^{\prime} 52^{\prime \prime}$ & $8^{\prime} 21^{\prime \prime}$ & $3^{\prime} 32^{\prime \prime}$ & $1^{\prime} 10^{\prime \prime}$ & 2,57 \\
\hline
\end{tabular}

Tabla 4. Repuesta de los suelos ante la lluvia. Tiempo de encharcamiento (tp), tiempo de escorrentía (te), tiempo de cierre de grietas (tgc), tiempo de inicio de la escorrentía en la salida de la parcela (inicio del hidrograma) (tes), tiempo de fin de la escorrentía tras el fin de la lluvia (tef) y precipitación necesaria para producirse la escorrentía (Pe).

Soil response to the rain. Time to ponding (tp), time to runoff (te), time to crack's close (tgc), time to runoff at the plot outlet (start of the hydrograph) (tes), time to the end of the runoff after the end of the rainfall (tef) and rainfall volume to start the runoff $(P e)$.

tante mayores en Anna 6,05 mm (2,9 - 9,99 mm). El tiempo necesario para la finalización de la escorrentía fue mayor en Petrer que en Anna, lo que está directamente relacionado con los mayores caudales generados en el primer caso (Tablas 3 y 4 ).

La tasa de infiltración final estable es mucho mayor en Anna: $38,63 \mathrm{~mm} \mathrm{~h}^{-1}$ (52,71 - 28,36 $\left.\mathrm{mm} \mathrm{h}^{-1}\right)$; que en Petrer: $11,16 \mathrm{~mm} \mathrm{~h}^{-1}\left(7,43-14,23 \mathrm{~mm} \mathrm{~h}^{-1}\right)$. Esto, junto a escorrentías generadas muy rápidamente en Petrer, explica tasas de escorrentía mayores que alcanzan coeficientes de escorrentías de $64,7 \%$ en 30 minutos $(58,7-72,9 \%)$ y $72,2 \%$ en 60 minutos $(66,4-79,7 \%)$. Por el contrario, en Anna, las escorrentías fueron muy reducidas. El coeficiente de escorrentía medio fue de $12 \%(1,1-23,5 \%)$ en 30 minutos de lluvias y de $19,4 \%$ $(2,6-31,7 \%)$ con chaparrones de 1 hora. En este comportamiento de los suelos de Anna (Tabla 5), además de la alta capacidad de infiltración final estable $\left(f c, 38,6 \mathrm{~mm} \mathrm{~h}^{-1}\right)$ es de destacar que la escorrentía se inició más tarde que en Petrer (tes, 8' 36"). 
PIRINEOS 149-150

\begin{tabular}{|crrr|}
\hline Parcelas & $\begin{array}{c}f c \\
\left(\mathrm{~mm} \mathrm{~h}^{-1}\right)\end{array}$ & \multicolumn{1}{c}{$\begin{array}{c}\text { Ce30 } \\
(\%)\end{array}$} & \multicolumn{1}{c|}{$\begin{array}{c}\text { Ce60 } \\
(\%)\end{array}$} \\
\hline ANNA & & & \\
A & 45,74 & 5,23 & 9,58 \\
B & 52,71 & 1,14 & 2,58 \\
C & 28,36 & 15,46 & 27,27 \\
D & 33,82 & 14,66 & 25,74 \\
E & 32,50 & 23,48 & 31,66 \\
Media & 38,63 & 11,99 & 19,37 \\
Std & 9,11 & 8,87 & 12,57 \\
Cv (\%) & 23,58 & 73,94 & 64,90 \\
\hline PETRER & & & $\cdot$ \\
A & 7,43 & 72,93 & 79,71 \\
B & 14,23 & 58,68 & 66,39 \\
C & 12,20 & 62,56 & 70,19 \\
D & 10,25 & 66,05 & 73,71 \\
E & 11,70 & 63,21 & 70,97 \\
Media & 11,16 & 64,69 & 72,19 \\
Std & 2,26 & 5,31 & 4,95 \\
Cv (\%) & 20,25 & 8,20 & 6,86 \\
\hline
\end{tabular}

Tabla 5. Hidrología de los suelos. Tasa de infiltración final estable (fc), coeficiente de escorrentía hasta el minuto 30 (Ce30) y el minuto 60 (Ce60).

Soil hydrology. Steady-state infiltration rate $(f c)$, runoff coefficient until the minute $30(\mathrm{Ce} 30)$, and until the minute 60 (Ce60).

\subsection{Pérdida de suelo}

La erodibilidad de los suelos es muy distinta entre Petrer y Anna. En Anna la concentración de sedimentos en la escorrentía es de $2,01 \mathrm{~g} \mathrm{l}^{-1}$, oscilando entre 1,36 y 2,69 $\mathrm{g} \mathrm{l}^{-1}$. En Petrer es mucho mayor: $57,63 \mathrm{~g} \mathrm{l}^{-1}$, variando en 25,75 y $82,23 \mathrm{~g} \mathrm{l}^{-1}$. Estas diferencias, junto a las que se daban en la hidrología presentadas anteriormente, explican tasas de erosión elevadísimas en Petrer $\left(2.198 \mathrm{~g} \mathrm{~m}^{2} \mathrm{~h}^{-1}\right)$ con variaciones espaciales importantes (3.221 - $848 \mathrm{~g}$ $\left.\mathrm{m}^{2} \mathrm{~h}^{-1}\right)$. En Anna, por el contrario, las tasas de erosión son mucho menores $\left(13 \mathrm{~g} \mathrm{~m}^{2} \mathrm{~h}^{-1}\right)$, aunque las variaciones son también elevadas $\left(0,85-23,7 \mathrm{~g} \mathrm{~m}^{2} \mathrm{~h}^{-1}\right)$ (Tabla 6).

Otra de las diferencias destacables en cuanto a la erosionabilidad de los badlands desarrollados sobre margas y arcillas reside en que la concentración de sedimentos aumenta a lo largo del experimento en Petrer $(+0,44)$ con una oscilación entre - 0,06 y $+0,82$, mientras que en Anna se produce una clara reducción con el tiempo de la erodibilidad de los suelos: $-0,08$ (entre $-0,02$ y $-0,22)$. 
INFLUENCIA DE LA LITOLOGÍA EN LOS PROCESOS DE EROSIÓN EN BADLANDS

\begin{tabular}{|c|c|c|c|c|c|}
\hline Parcelas & $\begin{array}{c}C s \\
\left(\mathrm{~g} \mathrm{l}^{-1}\right) \\
\end{array}$ & $\begin{array}{c}T e \\
\left(\mathrm{~g} \mathrm{~m}^{2} \mathrm{~h}^{-1}\right) \\
\end{array}$ & $T C S$ & $\begin{array}{c}S a \\
(\mu s)\end{array}$ & $T S a$ \\
\hline \multicolumn{6}{|l|}{ ANNA } \\
\hline A & 2,69 & 7,50 & $-0,22$ & 1056 & $-16,25$ \\
\hline B & 1,36 & 0,85 & $-0,05$ & 867 & $-8,31$ \\
\hline C & 1,98 & 15,35 & $-0,04$ & 1324 & $-3,14$ \\
\hline $\mathrm{D}$ & 2,19 & 17,46 & $-0,07$ & 1236 & $-15,24$ \\
\hline $\mathrm{E}$ & 1,83 & 23,67 & $-0,02$ & 920 & $-14,35$ \\
\hline Media & 2,01 & 12,97 & $-0,08$ & 1080,55 & $-11,46$ \\
\hline Std & 0,43 & 7,97 & 0,07 & 176,38 & 4,99 \\
\hline $\mathrm{Cv}(\%)$ & 21,57 & 61,43 & $-89,54$ & 16,32 & $-43,58$ \\
\hline \multicolumn{6}{|l|}{ PETRER } \\
\hline A & 82,23 & 3220,57 & $-0,06$ & 159 & $-9,39$ \\
\hline B & 25,75 & 847,85 & 0,36 & 150 & $-3,25$ \\
\hline C & 55,16 & 1991,47 & 0,74 & 129 & $-5,87$ \\
\hline $\mathrm{D}$ & 56,15 & 2136,59 & 0,82 & 201 & $-9,57$ \\
\hline $\mathrm{E}$ & 68,88 & 2792,01 & 0,36 & 124 & $-4,25$ \\
\hline Media & 57,63 & 2197,70 & 0,44 & 152,60 & $-6,47$ \\
\hline Std & 18,74 & 808,43 & 0,32 & 27,44 & 2,60 \\
\hline $\mathrm{Cv}(\%)$ & 32,52 & 36,79 & 71,04 & 17,98 & $-40,21$ \\
\hline
\end{tabular}

Tabla 6. Pérdida de suelo. Concentración de sedimentos (Cs), tasa de erosión (Te), tendencia de la concentración de sedimentos (Tcs), salinidad de la escorrentía (Sa) y tendencia de la salinidad (TSa).

Soil loss. Sediment concentration (Cs), erosion rates (Te), trends of the sediment concentration (Tcs), runoff salinity $(\mathrm{Sa})$ and trends of the runoff salinity $(\mathrm{TS} a)$.

La pérdida de suelo, además de por el arrastre de sedimentos, se produce por la exportación de solutos, lo que es cuantificable a partir de la salinidad de la escorrentía mediante la medición de la conductividad eléctrica. Contrariamente a la producción de agua y sedimentos, la mayor salinidad de la escorrentía se ha observado en Anna. Allí, se alcanzaron $1.081 \mu \mathrm{S}$ (1.236 $867 \mu S)$, mientras que en Petrer fue de $153 \mu S(124-201 \mu S)$ por término medio. La salinidad de la escorrentía se reduce durante la lluvia en ambas zonas de estudio: - 11,5 en Anna y -6,5 en Petrer.

\section{Discusión}

Los badlands sobre arcillas del Keuper presentan una capa de regolita más profunda, menos densa y con grietas mayores que en las margas, aunque con menor densidad. También la textura arcillosa y la estructura es más estable en 
las arcillas, lo que influye sobre la respuesta hidrológica y erosiva ante la lluvia de estos dos tipos de badlands. Estos condicionantes explican encharcamientos, escorrentías y cierre de grietas muy rápidos en las margas, y con ello pérdidas de suelo y tasas de escorrentía mayores que en los badlands sobre arcillas.

En el caso de los badlands sobre margas, las tasas de erosión elevadas se han confirmado en distintas zonas del planeta como Israel (YAIR et al., 1980), Túnez (BERNDTSSON, 1988), Marruecos, (IMESON, 1983), Italia (ALEXANDER, 1982), Estados Unidos (PARCKER \& SCHUMM, 1982) o Canada (CAMPBELL, 1974), así como en la Península Ibérica, donde aparecen repetidamente en los paisajes del sudeste (CALVO et al., 1992), en las dos mesetas y en la depresión del Ebro (BENITO et al., 1991). La presencia de badlands en zonas húmedas es menos importante, aunque existen, como lo demuestra su presencia en zonas como los Pirineos (REGÜÉS, 1995). Escorrentías y tasas de erosión reducidas se han medido en otras zonas del País Valenciano en badlands desarrollados sobre arcillas (CERDÀ \& PAYÀ, 1995). aunque es lo menos habitual.

La arroyada superficial es el principal agente geomórfico en las zonas de badlands, si bien las tubificaciones y los movimientos en masa también se produce en algunos casos (HARVEY, 1982; BENITO et al., 1991). Esta afirmación es mucho más evidente cuando el roquedo es más impermeable y erosionable como son las margas. En el caso de las arcillas, las tasas de erosión y escorrentía son semejantes a las encontradas en otros ambientes del sudeste peninsular donde la vegetación es mayor (CERDÀ, 1994-1995).

Normalmente, la concentración de sedimentos en la escorrentía decrece con el tiempo debido al agotamiento del material susceptible de ser erosionado (ABRAHAMS et al., 1988). Este comportamiento se ha encontrado en las arcillas, pero en las margas el aumento de la concentración de sedimentos indica la existencia de un control químico del proceso de erosión en estos badlands. La dispersión de los agregados al entrar en contacto con el agua determina un aumento de la erodibilidad del suelo con el tiempo lo que se ha confirmado en estudios sobre la estabilidad de los agregados (CERDÀ, 1993) y en investigaciones similares a la presentada en el sur de la Península Ibérica (IMESON \& VERSTRATEN, 1988; GERITS, 1991).

Las implicaciones geomorfológicas de este comportamiento hidrológico y erosivo de los badlands son el que en las margas se producirá un importante aumento de las tasas de erosión con el tiempo debido a que el suministro de sedimentos no se agota sino que aumenta. Esto significa que los procesos de estabilización en los badlands sobre margas difícilmente se producirán, al contrario que en las arcillas donde las tasas de erosión y las escorrentías son menores y se reducen con el agotamiento de los sedimentos exportables. 
La Figura 6 ilustra las diferencias entre las dos zonas estudiadas. La relación del coeficiente de escorrentía con la concentración de sedimentos y la tasa de escorrentía demuestra que siempre los badlands de Anna tienen menores pérdidas de agua y suelo durante las lluvias, mientras que en el caso de Petrer la actividad erosiva es muy eficiente.

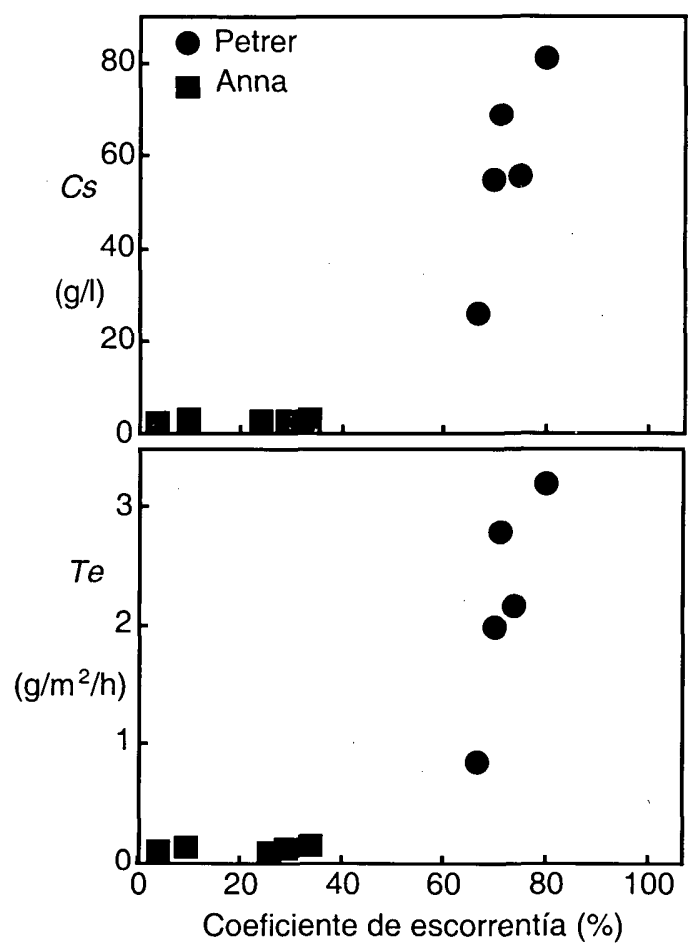

Figura 6. Relación del coeficiente de escorrentía ( \%) con la concentración de sedimentos (Cs) y la tasa de erosión (Te) para las dos zonas de estudio: Petrer y Anna.

Relationship of the runoff coefficient (\%) with the sediment concentration (Cs) and the erosion rate (Te) for the two study zones: Petrer and Anna.

\section{Conclusiones}

Las zonas degradadas o badlands presentan un comportamiento erosivo e hidrológico muy distinto dependiendo de la litología. En las arcillas del Keuper el encharcamiento y la escorrentía son retrasados, las tasas de infiltración elevadas y las tasas de escorrentía y erosión reducidas. En cambio, sobre margas, 
el encharcamiento y la escorrentía superficial se produce casi inmediatamente, las tasas de infiltración son muy bajas y las tasas de escorrentía y erosión elevadísimas. La actividad de la arroyada superficial en las margas hace que las posibilidades de su regeneración sean menores que en las arcillas debido a las elevadas pérdidas de suelo y agua. Los resultados de este estudio demuestran que en las zonas áridas, donde la vegetación es reducida, la litología puede jugar un papel relevante a la hora de determinar los procesos de erosión.

Agradecimientos. Se agradece a la Conselleria de Educació y Ciència (Generalitat Valenciana) la beca que permitió la realización de estos experimentos y al Ministerio de Educación y Ciencia la concesión de una beca postdoctoral en el extranjero entre 1994 y 1996. Agradezco a Mario Payà y a toda su familia la ayuda prestada durante la realización del trabajo de campo y a la Comisión Interministerial de Ciencia y Tecnología la financiación del trabajo de campo en Petrer (proyectos AMB93-0844-C06-04 y NAT91-0741). La revisión final del manuscrito se realizó bajo la financiación del proyecto CLI95-1890 y el Ministerio de Educación y Ciencia mediante un contrato de incorporación de doctores y tecnólogos. Dos evaluadores anónimos incorporaron interesantes mejoras al primer manuscrito.

\section{Referencias}

ABRAHAMS, A.D., PARSONS, A.J. \& LUK, S.H. (1988): Hydrologic and sediment response to simulated rainfall on desert hillslopes in southern Arizona. Catena, 15: 103-117.

ALEXANDER, D. (1982): Difference between «calanchi» and «biancane» badlands in Italy. En BRYAN, R. Y YAIR, A. (Eds.): Badland geomorphology and piping: 71-88. Norwinch.

ARNÁEZ VADILLO, J. (1988): Rills, gullies and badlands: Reviewing the problem. Pirineos, 132: 89-98.

BENITO, G., GUTIÉRREZ. M. \& SANCHO, C. (1991): Erosion patterns in rill and interill areas in badlands zones of the middle Ebro basin (N.E-Spain). En SALA, M., RUBIO, J.L. \& GARCÍA-RUIZ, J.M. (Eds.): Soil Erosion Studies in Spain: 41-54. Logroño.

BERNDTSSON, R. (1988): Spatial hydrological processes in a water resources planning perspective. An investigation of rainfall and infiltration in Tunisia. University of Lund. Report n. ${ }^{\circ} 1009.315$ pp. 
INFLUENCIA DE LA LITOLOGIAA EN LOS PROCESOS DE EROSIÓN EN BADLANDS

BERNDTSSON, R., HOGLAND, W. \& LARSON, M. (1985): Spatial variations of infiltration for a catchment in northern Tunisia. En BERNDTSSON, R., LARSON, M., DAHLBLOM, P., NGUYEN, V.L., HOGLAND, W. \& NIEMCZYNOWICZ, J. (Eds.): Hydrological studies in Tunisia: 13-26. Lund.

BRYAN, R. \& YAIR, A. (Eds.) (1982): Badland geomorphology and piping. Goebooks, Norwich, 409 pp.

CALVO, A., HARVEY, A. \& PAYÀ, J. (1992): Response of badland surfaces in south east Spain to simulated rainfall. Cuaternario y Geomorfologia, 6: 3-14.

CAMPBELL, I.A. (1974): Erosion rates in the Steveville badlands. Alberta. Z. Geomorph. N. F. Suppl.-Bd., 21: 122-137.

CAMPBELL, I.A. (1989): Badland and Badland Gullies. En THOMAS, D.S.G. (Ed.): Arid zone geomorphology: 159-186. London.

CERDÀ, A. (1993): Estabilidad de agregados en suelos degradados. País Valenciano. In Actas XIII Congreso de Geografía. Universidad de Sevilla. Nuevos Procesos Territoriales: 187-192. Sevilla.

CERDÀ, A. (1994-1995): Influencia de la exposición sobre la producción de sedimentos y escorrentías en ambientes semiáridos. Cuadernos de Investigación Geográfica, 20-21: 47-63.

CERDÀ, A., IBÁÑ̃EZ, S. \& CALVO, A. (1997): Design and operation of a small and portable rainfall simulator for rugged terrain. Soil Technology, 11: 161168.

CERDÀ, A. \& PAYÀ, J. (1995): Morfología y procesos superficiales en badlands sobre arcillas del Keuper, Requena, Valencia. Cuaternario y Geomorfología, 9: 59-72.

CLOTET, N., GALLART, F. \& SALA, M. (1987): Los badlands: características, interes teórico, dinámica y tasas de erosión. Notas de Geografía Física: 15-16.

ELÍAS, J. \& RUIZ, L. (1979): Precipitaciones máximas en España. Ministerio de Agricultura, Madrid, 545 pp.

GEIGER, F. (1970): Die Ariditat in Sudostspanien. Stuttgarter Geographische Studien, Band 77. Stuttgart.

GERITS, J.J.P. (1991): Physico-chemical tresholds for sediment detachment, transport and deposition. Universiteit van Amsterdam, Amsterdam, $186 \mathrm{pp}$.

HARVEY, A.M. (1982): The role of piping in the development of badlands and gully systems in south-east Spain. En BRYAN, R. \& YAIR, A. (Eds.): Badland geomorphology and piping: 317-336.

HOWARD, A.D. (1994): Badlands. En ABRAHAMS A.D. \& PARSONS, A.J. (Eds.): Geomorphology of Desert Environments: 213-242. Norwich.

IMESON, A.C. (1983): Studies of erosion tresholds in semi-arid areas: field measurement of soil loss and infiltration in northern Marocco. Catena Suppl-Bd., 4: 79-89. 
IMESON, A.C. \& VERSTRATEN, J.M. (1988). Rills on badland slopes: a physico-chemical controlled phenomenon. Catena Suppl.-Bd., 12: 139-53.

JORGENSEN, D.W. \& GARDNER, T.W. (1987): Infiltration capacity of disturbed soils: temporal change and lithologic control. Water Resources Bulletin, 23 (6): 1161-1172.

LÓPEZ BERMÚDEZ, F., RUBIO RECIO, J.M. \& CUADRAT, J.M. (1992): Geografía Física. Editorial Cátedra, 594 pp. Madrid.

PARCKER, R.S. \& SCHUMM, S.A. (1982): Experimental study of drainage networks. En BRYAN, R.B. Y YAIR, A. (Eds.): Badland Geomorphology and Piping: 153-168. Norwich.

PAYÀ, J. \& CERDÀ, A. (1992): Cambios morfológicos y respuesta a la lluvia simulada de tres superficies de badland. Petrer. Alacant. En LÓPEZ BERMÚDEZ, F., CONESA GARCÍA, C. \& ROMERO DÍAZ, M.A. (Eds.): Estudios de Geomorfología en España: 161-170. Murcia.

REGÜÉS, D. (1995): Meteorización física en relación con los procesos de producción y transporte de sedimentos en un área acarcavada. Tesis Doctoral inédita, Institut de Ciències de la Terra Jaume Almera, (CSIC). Facultat de Ciències Geològiques (Universitat de Barcelona), Barcelona, $281 \mathrm{pp}$.

RODRÍGUEZ, J. (1992): Un ejemplo de la influencia del uso y abandono de bancales de fondo de canal en la evolución de badlands (Petrer-Alacant). En LÓPEZ BERMÚDEZ, F., CONESA GARCÍA, C. \& ROMERO DÍAz, M.A. (Eds.): Estudios de Geomorfología en España: 211-220. Murcia.

SANROQUE, P., RUBIO, J.L. \& IZQUIERDO, L. (1990): Relationship among erodibility, parent material and soil type in areas of the Valencia province (Spain). Soil Technology, 3: 373-384.

SUMMERFIELD, M.A. (1991): Global Geomorphology. Logman Scientific and technical, Essex, Inglaterra, $537 \mathrm{pp}$.

YAIR, A., LAVEE, H., BRYAN, R.B. \& ADAR, E. (1980): Runoff and erosion processes and rates in the Zin Valley Badlands. Northern Negev. Israel. Earth Surface Processes and Landforms, 5: 205-225. 